\title{
Multisystem Disorder in Late-Onset Chronic Progressive External Ophthalmoplegia
}

\author{
Gerald Pfeffer, Sandra Sirrs, N. Kevin Wade, Michelle M. Mezei
}

\begin{abstract}
Introduction: Chronic progressive external ophthalmoplegia (CPEO) is a mitochondrial syndrome on a disease spectrum with Kearns-Sayre syndrome (KSS). Clinical presentation is variable and our experience suggested that phenotypic differences exist in CPEO with onset after age 20. Methods: This descriptive study is a retrospective chart review of 40 patients with late-onset CPEO. Clinical features, laboratory and neurophysiology results were reviewed. Results: Multisystem dysfunction was very common in this series. Gastrointestinal dysfunction was more common than expected $(60 \%)$ as was migraine headache (40\%). Clinical characteristics on the KSS disease spectrum were uncommon in this series with only $2.5 \%$ having pigmentary retinopathy, $5 \%$ with cardiac conduction abnormality, and $22.5 \%$ having endocrinopathy (most often thyroid dysfunction rather than diabetes). Neurophysiology abnormalities included length-dependent axonal polyneuropathy in 44\% (sometimes subclinical) and myopathic EMG changes in $26 \%$. Exposure to sources of acquired mitochondrial toxicity including cigarette use and hepatitis $\mathrm{C}$ infection were more common than expected in this series. Discussion: Phenotype was different in this late-onset series compared with previous reports in CPEO patients. In this series of late-onset patients, multi-organ dysfunction was more common than previously reported in CPEO, and some classical mitochondrial manifestations, such as pigmentary retinopathy were rare. We suggest that acquired mitochondrial toxicity may have a role in the pathogenesis of adult-onset CPEO.
\end{abstract}

RÉSUMÉ: Maladie multisystémique dans l'ophtalmoplégie externe progressive chronique à début tardif. Contexte : L'ophtalmoplégie externe progressive chronique (OEPC) est un syndrome mitochondrial qui se situe dans le spectre de maladies dont fait partie le syndrome de Kearns-Sayre (SKS). Sa présentation clinique est variable et, selon notre expérience, nous croyons qu'il existe des différences phénotypiques dans l'OEPC qui commence après l'âge de 20 ans. Méthodes : Il s'agit d'une étude descriptive de 40 patients atteints d'OEPC à début tardif dont nous avons révisé rétrospectivement les dossiers pour en tirer les observations cliniques, les données de laboratoire et les résultats d'études neurophysiologiques. Résultats : La dysfonction multisystémique était très fréquente chez ces patients. La dysfonction gastrointestinale (60\%) et la migraine (40\%) étaient plus fréquentes qu'attendu. Les caractéristiques cliniques du spectre du SKS étaient rares dans cette série de patients, seulement 2,5\% étant porteurs d'une rétinopathie pigmentaire, $5 \%$ d'anomalies de conduction cardiaque et 22,5\% d'endocrinopathies (la plupart du temps une dysfonction thyroïdienne plutôt qu'un diabète). Parmi les anomalies neurophysiologiques, nous avons retrouvé une polyneuropathie axonale longueur-dépendante chez 44\% (parfois subclinique) et des changements de type myopathique à l'EMG chez 26\%. Une exposition à des sources de toxicité mitochondriale acquise dont le tabagisme et l'infection par le virus de l'hépatite C était plus fréquente qu'attendu dans cette série de cas. Discussion : Le phénotype était différent chez nos cas à début tardif comparé à celui décrit dans les études publiées antérieurement. Chez nos patients atteints d'OEPC à début tardif, une dysfonction multisystémique était plus fréquente que chez les cas publiés antérieurement et certaines manifestations mitochondriales classiques telle une rétinopathie pigmentaire étaient rares. Nous proposons qu'une toxicité mitochondriale acquise puisse jouer un rôle dans la pathogenèse de l'OEPC débutant à l'âge adulte.

Can. J. Neurol. Sci. 2011; 38: 119-123

Chronic progressive external ophthalmoplegia (CPEO) is a mitochondrial syndrome characterized by slowly progressive bilateral ptosis and ophthalmoparesis. Patients with CPEO have variable presentations ranging from pure CPEO to a CPEO "plus" syndrome with other accompanying multisystem features of mitochondrial disease. Classically these additional features include skeletal myopathy, pigmentary retinopathy (PR) and endocrine dysfunction. ${ }^{1}$ These features place CPEO on a disease spectrum with Kearns-Sayre syndrome (KSS), another mitochondrial deletion syndrome characterized by CPEO, PR and onset earlier than 20 years-of-age, and frequently accompanied by other features of mitochondrial disease. ${ }^{2}$
Mitochondrial diseases are often difficult to diagnose because of their highly variable presentation and the absence of reliable non-invasive tests. These diagnostic challenges and variability in

\footnotetext{
From the Division of Neurology (GP, MMM), Division of Endocrinology (SS), Department of Ophthalmology and Visual Sciences (NKW), University of British Columbia; Adult Metabolic Disease Clinic (SS, MMM), Vancouver General Hospital, Vancouver, British Columbia, Canada.

Received May 14, 2010. Final Revisions Submitted June 29, 2010. Correspondence to: Michelle Mezei, Adult Metabolic Diseases Clinic, 4th Floor, 2775 Laurel St., Vancouver, British Columbia, V5Z 1M9, Canada.
} 
clinical presentation prompted this case series to further describe the clinical spectrum of the disorder in a population of patients with late-onset CPEO. Experience in our clinic suggested that the burden of multi-system manifestations of mitochondrial disease was high.

\section{Methods}

This study is a retrospective chart review of patients referred to the Adult Metabolic Diseases Clinic in Vancouver, Canada from 2000 until 2008. Research ethics board approval was obtained. For inclusion patients were over 20 years-of-age with a diagnosis of CPEO made by two of the authors who are specialists in neuromuscular and metabolic diseases. For the purposes of this study, late-onset CPEO was arbitrarily defined as a patient with CPEO whose earliest symptoms of disease started after the age of 20. Diagnosis required characteristic clinical features (presence of slowly progressive ophthalmoparesis and ptosis in the absence of symptoms or signs of another aetiology) and was supported by muscle pathology and/or molecular genetic studies. Genetic testing included longrange polymerase chain reaction (PCR) with confirmation on Southern blot, and testing for other mitochondrial DNA point mutations (the panel includes A8344G (typically associated with MERRF), A3242G, T3721C and T3721deln (typically associated with MELAS)). Testing for nuclear DNA mutations associated with CPEO was requested in selected cases.

Findings on ocular examination were confirmed by a neuroophthalmologist. Laboratory testing to support the diagnosis of mitochondrial disease was obtained including pre- and postprandial serum lactate, urine organic acid profile, and acylcarnitine profile. Alternative etiologies were excluded when appropriate with bloodwork (AChR antibody testing and oculopharyngeal muscular dystrophy DNA analysis), neurophysiology (electromyography (EMG), nerve conduction studies (NCS), single fiber EMG, and repetitive nerve stimulation) and neuroimaging (CT, and/or MR imaging of head and orbits, MR spectroscopy). Patients had screening for systemic complications of mitochondrial disease including endocrine and cardiac dysfunction, and when indicated by symptoms, screening of auditory and gastrointestinal systems.

Charts were identified for review from the clinic database, by searching for patients with a diagnosis of CPEO. Initially 45 subjects were identified, but five subjects were excluded because they did not meet entry criteria (two patients with fibre-type disproportion myopathy, and three patients with HIV and CPEO-

Table 1: Age and sex of subjects with CPEO

\begin{tabular}{l|c}
\hline Age (range) & $54.5(27-74)$ \\
\hline Sex M:F & $14: 26$ \\
\hline Average age at diagnosis & 50.8 \\
\hline $\begin{array}{l}\text { Average symptom duration pre- } \\
\text { diagnosis (range) }\end{array}$ & $11.8(1-50)$ \\
\hline $\begin{array}{l}\text { Average number of multisystem } \\
\text { manifestations (range) }\end{array}$ & $4.6(0-10)$ \\
\hline
\end{tabular}

like syndromes on antiretrovirals, who were reported separately ${ }^{3}$ ). The remaining 40 charts were reviewed, to document demographic characteristics, age at diagnosis, age of onset of symptoms, and multisystem manifestations. The age of symptom onset was determined based on patients' and/or their families' self-report. The presence of ptosis, ophthalmoparesis, diplopia, PR and optic atrophy was recorded and confirmed by the neuro-ophthalmologist. Other possible neurological and systemic manifestations of mitochondrial disease were tabulated including: skeletal myopathy, exertional myalgias, fatigue, peripheral neuropathy, migraine headaches, seizures, stroke-like episodes, ataxia, sensorineural hearing loss, neuropsychiatric disorder, gastrointestinal disorder, cardiac or endocrine involvement. Ascertainment of more subjective complaints such as fatigue and neuropsychiatric symptoms of depressed mood and poor memory may not have been complete though when available psychiatric consultations or neuropsychology cognitive assessments were reviewed. Blood work indicated the presence of endocrinopathy (thyroid stimulating hormone [TSH], fasting glucose, serum and urine osmolality for diabetes insipidus), liver or renal involvement. Diagnostic tests confirmed polyneuropathy, skeletal myopathy (EMG/NCS), sensorineural hearing loss (audiometry) and cardiac disorder (ECG/echocardiogram/Holter monitor). Reports of neuroimaging studies, including CT and MRI scans of the head, were reviewed. All of the above clinical features were questioned in every initial consultation. The prevalence of cigarette use, alcohol abuse, or chronic viral infection (HIV and hepatitis $\mathrm{C}$ virus $[\mathrm{HCV}]$ ) were documented.

\section{RESULTS}

Refer to Table 1 for details regarding patient characteristics and Table 2 for multi-organ manifestations of mitochondrial disease. All of the subjects were from different families, except for one sibling pair. Diplopia was only intermittent and mild but found in 25/40 (62.5\%) of patients. Skeletal muscle weakness preferentially affected the proximal musculature, and often coexisted with exertional myalgia. One patient had chest wall weakness and exertional dyspnoea. Gastrointestinal disorder present in 24 patients $(60 \%)$ was most commonly dysphagia $(18 / 24)$, followed by diarrhoea (5/24) and constipation $(1 / 24)$. Subjective complaints of fatigue and neuropsychiatric disorders (including depression, bipolar illness and cognitive dysfunction) were common ( $42.5 \%$ and $37.5 \%$, respectively), and coexisted in $9(22.5 \%)$. Endocrine disorders $(22.5 \%)$ were most frequently hypothyroidism (6/9), followed by diabetes mellitus (2/9) and one patient had multiple endocrine abnormalities, including diabetes insipidus, growth hormone deficiency and adrenal insufficiency. Pigmentary retinopathy was only found in one patient $(2.5 \%)$. Cigarette use was documented in $20(50 \%)$, alcohol abuse in two $(5 \%)$ and chronic viral infections in four (10\%, all HCV).

Laboratory investigations revealed that 11 (27.5\%) patients had elevated serum lactate. Of the 39 patients who had EMG/NCS, 17 (44\%) had an axonal sensory polyneuropathy which was subclinical in four patients, and was a sensorimotor neuropathy in three patients. Myopathic changes were present in ten patients $(26 \%)$, and mild nonspecific EMG abnormalities were present in three. Sixteen patients $(41 \%)$ had normal 
EMG/NCS. Muscle biopsy was performed in 36 patients. Four patients did not have muscle biopsy because of an already confirmed diagnosis on genetic testing (two cases) or confirmed diagnosis in a direct relative (two cases). Characteristic findings of mitochondrial myopathy were present on histopathology in $26 / 36$ patients $(72 \%)$ and on electron microscopy (EM) in $17 / 26$ $(65 \%)$. Of the ten patients with nondiagnostic histopathology, four had findings on EM characteristic of mitochondrial disease. Genetic studies demonstrated the presence of single large-scale mtDNA deletions in 11/26 (42\%) and nuclear mutations in three patients (all PolG1 mutations, including 1. Heterozygous T251I and P587L mutations; 2. heterozygous N1098I mutation; 3. Heterozygous A467T and G737R mutations (this patient reported previously in Milone et $\mathrm{al}^{4}$ ). Three of the patients with pathogenic genetic abnormalities had normal muscle histopathology (two with PolG1 mutations, and one with a $3.8 \mathrm{~kb}$ mtDNA deletion involving $70 \%$ of genomes). In total, the number of patients in this series with diagnosis proven by muscle pathology and/or genetic abnormalities was 35 (87.5\%). Respiratory chain enzyme analysis performed on 12 of the biopsies provided diagnostic criteria in only one patient. Acylcarnitine and urine organic acid profiles were normal in all patients. Magnetic resonance imaging revealed non-specific white matter abnormalities in nine patients and thin extraocular muscles in three patients.

\section{DISCUSSION}

To our knowledge this series is the first of its kind to be directed specifically toward the study of clinical features of

Table 2: Prevalence of manifestations of CPEO and other features of mitochondrial disease

\begin{tabular}{l|c|c}
\hline Symptom/sign & Number & Percentage \\
\hline Ophthalmoplegia & 40 & 100 \\
\hline Ptosis & 40 & 100 \\
\hline Diplopia (intermittent) & 25 & 62.5 \\
\hline $\begin{array}{l}\text { Skeletal muscle weakness (on } \\
\text { examination) }\end{array}$ & 25 & 62.5 \\
\hline Gastrointestinal disorder & 24 & 60 \\
\hline Sensorineural hearing loss & 19 & 47.5 \\
\hline Fatigue & 17 & 42.5 \\
\hline Migraine & 16 & 40 \\
\hline Neuropsychiatric disorder & 15 & 37.5 \\
\hline Exertional myalgia & 14 & 35 \\
\hline Polyneuropathy (clinically and & 13 & 32.5 \\
confirmed on NCS) & & \\
\hline Endocrinopathy $\quad$ Hypothyroidism & 9 & 22.5 \\
\multicolumn{1}{c|}{ Diabetes mellitus (type II) } & 2 & 15 \\
$\quad$ Multiple & 1 & 5 \\
\hline Ataxia & 7 & 2.5 \\
\hline Cardiomyopathy & 3 & 17.5 \\
\hline Cardiac conduction abnormality & 2 & 5 \\
\hline Hemiparesis/stroke-like episodes & 2 & 5 \\
\hline Optic atrophy & 2 & 5 \\
\hline Pigmentary retinopathy & 1 & 2.5 \\
\hline
\end{tabular}

CPEO patients with onset later than 20 years-of-age. This series reveals a number of differences in comparison with previously reported multisystem dysfunction in CPEO (Table 3). Pigmentary retinopathy was rare in our series, although the prevalence appears to be high in earlier-onset CPEO. ${ }^{1,5,6}$ Much lower rates have also been demonstrated although patients with earlier age of onset appeared more likely to have PR, ${ }^{7}$ and two other studies including patients from wide age ranges did not specify age for patients with PR. ${ }^{8,9}$ Our patient with PR had disease onset at age 20, with sensorineural hearing loss.

The frequent finding of intermittent diplopia suggests that the presence of diplopia should not exclude a slowly progressive disorder of ocular motility such as CPEO, as has been suggested previously. ${ }^{10}$ Endocrine disorder was less common than previously reported. The endocrine disturbance classically described with mitochondrial disease (and in particular KSS) is diabetes, although thyroid dysfunction is also well described. ${ }^{11}$ In our series the majority of patients with endocrine disturbance had hypothyroidism. Gastrointestinal disorder occurred in $60 \%$, and the large majority of these patients had dysphagia. Dysphagia was reported in $75 \%$ from a series of CPEO and KSS patients, ${ }^{12}$ and in $30 \%$ of patients from another series of CPEO patients. ${ }^{7}$ Gastrointestinal manifestations are difficult to diagnose and probably under-recognized, but are potentially treatable. ${ }^{12}$ The very frequent occurrence of migraine is another feature distinguishing this series from previously published series on CPEO and KSS. The high female to male ratio in this study has been reported in prior series with $\mathrm{CPEO}^{13,14}$ and is of undetermined importance, but may relate to variable penetrance or expressivity between sexes (as is seen in Leber hereditary optic neuropathy, another mitochondrial syndrome), or perhaps variable susceptibility to mitochondrial toxicity based on the effects of sex hormones. ${ }^{15}$ Some of the documented features such as fatigue and neuropsychiatric disorders were very common and would have benefited from more objective measurement.

Previous series of CPEO demonstrating high prevalence of KSS-like features have likely focused on earlier-onset patients, who may have more in common with KSS patients than lateonset CPEO patients. As examples of this, one series found that $33 \%$ of CPEO patients had elevated CSF protein, ${ }^{9}$ which is one of the diagnostic criteria for KSS. Furthermore, another study found that 11/22 CPEO patients with mtDNA deletions had PR, but only $1 / 10$ patients without mtDNA deletions had PR, ${ }^{6}$ which is of interest given that KSS is a mtDNA deletion syndrome (and suggests that CPEO patients with PR are on a disease spectrum with KSS). In this series, late-onset CPEO patients appear to have a different constellation of multisystem dysfunction, with a more benign disease course and delayed onset of symptoms. We speculate this may be due to patients having subclinical mitochondrial genetic abnormalities (either from belowthreshold heteroplasmy or variable penetrance) that are expressed phenotypically after acquired mitochondrial toxicity from ageing or environmental exposures.

Electron microscopy analysis demonstrated similar yield to histopathology, although four patients with diagnostic findings on EM had nondiagnostic histopathology. Ultrastructural analysis should therefore be pursued even in the presence of nondiagnostic histopathology as it may provide additional criteria for diagnosis in some cases. The occasional difference in diagnostic findings with histopathology and electron microscopy 
Table 3: Prevalence of multisystem manifestations of mitochondrial disease compared with prior study

\begin{tabular}{|c|c|c|c|c|c|c|}
\hline & $\begin{array}{l}\text { Present } \\
\text { study (\%) }\end{array}$ & $\begin{array}{l}\text { Yamashita } \\
2008^{9}(\%)\end{array}$ & $\begin{array}{l}\text { Bau } \\
2005^{1}(\%)\end{array}$ & $\begin{array}{l}\text { Sciacco } \\
2001^{8}(\%)\end{array}$ & $\begin{array}{l}\text { Laforet } \\
1995^{7}(\%)\end{array}$ & $\begin{array}{l}\text { Holt } \\
1989^{6}(\%)\end{array}$ \\
\hline GI disorder & 60 & & & & 30 & \\
\hline Polyneuropathy & 44 & & 23 & & 21 & \\
\hline Migraine & 40 & & & & 21 & \\
\hline $\begin{array}{l}\text { Pigmentary } \\
\text { retinopathy }\end{array}$ & 2.5 & 7 & 65 & 9 & 16 & 38 \\
\hline $\begin{array}{l}\text { Endocrinopathy } \\
\text { Hypothyroidism } \\
\text { Diabetes mellitus }\end{array}$ & $\begin{array}{l}22.5 \\
15 \\
5 \\
\end{array}$ & & $\begin{array}{l}67 \\
\text { N/A } \\
\text { N/A }\end{array}$ & $\begin{array}{l}\text { N/A } \\
\text { N/A } \\
7^{*}\end{array}$ & & \\
\hline $\begin{array}{l}\text { Cardiac conduction } \\
\text { abnormality }\end{array}$ & 5 & 10 & 26 & & 19 & \\
\hline Skeletal myopathy & 62.5 & 81 & & 87 & 81 & \\
\hline
\end{tabular}

is likely the effect of genetic mosaicism which appears to be quite complex in these conditions. Prior study has demonstrated genotype differences between and within individual muscle fibres within the same specimen. ${ }^{16}$ There may also be differences in sensitivity or specificity for electron microscopy in mitochondrial disease.

In this series mtDNA deletions were the most common genetic abnormality, which is consistent with prior reports. ${ }^{7}$ The relatively low yield of molecular genetic analysis in this series is likely explained by the limited panel of mtDNA and nuclear DNA mutations tested. Despite this limitation, three patients with positive genetic studies (two PolG1 mutations and one mtDNA deletion) had nondiagnostic muscle biopsy results. The finding of a nondiagnostic muscle biopsy with a large scale mtDNA deletion in CPEO has been reported once previously ${ }^{17}$ and emphasizes that genetic studies should always be included in the workup of mitochondrial disease.

The finding of thin extraocular muscles in some patients on $\mathrm{CT}$ and MRI may be under-reported in this series, given that only the radiology reports were reviewed. This abnormality has been studied previously in CPEO, ${ }^{18,19}$ and therefore may be useful in the workup of suspected cases, although the specificity of this finding remains unknown.

Ophthalmoplegia and ptosis have been described in association with antiretrovirals ${ }^{3,20}$ and statin medication. ${ }^{21}$ Presumably the cause is acquired mitochondrial toxicity relating to these agents. In a series of patients with HIV and antiretrovirals apparently causing CPEO, the phenotype appears to be quite similar to those in this series. ${ }^{3}$ Acquired mitochondrial toxicity may also play a role in the expressivity of Leber hereditary optic neuropathy. ${ }^{22}$ It is possible that other unidentified sources of acquired mitochondrial toxicity may be in part responsible for late-onset CPEO, potentially explaining phenotypic differences with early-onset CPEO, and this should be further investigated. In this series, sources of acquired mitochondrial toxicity such as cigarette smoking ${ }^{23}$ and $\mathrm{HCV}$ infection $^{24}$ were more prevalent than expected (prevalence for British Columbia is $20.2 \%$ for smoking ${ }^{25}$ and $0.8 \%$ for $\mathrm{HCV}^{26}$ ). Remote alcohol abuse was documented in two patients. The importance of these results is speculative and requires validation in larger series with quantification of the severity of exposures.

Limitations of this study include the fact that the age for symptom onset for adult-onset CPEO was arbitrarily chosen at 20 years. This age was chosen for the practical reason that adult neurologists are most likely to see this condition in patients with adult-onset of symptoms, as well as to exclude patients with KSS and improve the homogeneity of our study population. However, age of onset was based upon patients' self-report and therefore reporting of age of onset may not be fully reliable and it is possible some patients had onset earlier than age 20 . Furthermore, it should be noted that not all patients had findings diagnostic for mitochondrial disease on muscle biopsy. We decided to include these patients in the review because in the practical sense, these patients do have a diagnosis of CPEO given their clinical syndrome and absence of alternative diagnoses after extensive investigation. It is well-known that definitive abnormalities on muscle biopsy are not present in all patients with mitochondrial disease, which may be related to heteroplasmy within tissues or the sampling of unaffected muscles. ${ }^{16}$ The alternative, which was to include only patients with diagnostic muscle biopsies, may have biased the findings away from the types of cases that would typically present to a clinical neurologist. Many patients did not have proven molecular abnormalities which was expected because of our limited panel available for point mtDNA mutations or nuclear DNA mutations.

\section{Conclusions}

In summary, this series presents the first review focusing exclusively on adult-onset CPEO patients. We have demonstrated phenotypic differences with prior series, and we suggest that acquired mitochondrial toxicity may interact with genetic abnormalities in the pathogenesis of adult-onset CPEO. 


\section{COMPETING INTERESTS}

Dr. Sirrs has received speaking fees from Shire Human Genetics Therapies, and is an investigator in the Canadian Fabry Disease Initiative which receives partial funding from Shire Human Genetics Therapies and Genzyme Corporation.

\section{REFERENCES}

1. Bau V, Zierz S. Update on chronic progressive external ophthalmoplegia. Strabismus. 2005;13(3):133-42.

2. Shoffner JM. Mitochondrial myopathy diagnosis. Neurol Clin. 2000;18(1):105-23.

3. Pfeffer G, Cote HC, Montaner JS, Li CC, Jitratkosol M, Mezei MM. Ophthalmoplegia and ptosis: mitochondrial toxicity in patients receiving HIV therapy. Neurology. 2009;73(1):71-2.

4. Milone M, Brunetti-Pierri N, Tang LY, et al. Sensory ataxic neuropathy with ophthalmoparesis caused by POLG mutations. Neuromuscul Disord. 2008;18(8):626-32.

5. Isashiki $\mathrm{Y}$, Nakagawa $\mathrm{M}, \mathrm{Ohba} \mathrm{N}$, et al. Retinal manifestations in mitochondrial diseases associated with mitochondrial DNA mutation. Acta Ophthalmol Scand. 1998;76(1):6-13.

6. Holt IJ, Harding AE, Cooper JM, et al. Mitochondrial myopathies: clinical and biochemical features of 30 patients with major deletions of muscle mitochondrial DNA. Ann Neurol. 1989;26 (6):699-708

7. Laforet P, Lombes A, Eymard B, et al. Chronic progressive external ophthalmoplegia with ragged-red fibers: clinical, morphological and genetic investigations in 43 patients. Neuromuscul Disord. 1995;5(5):399-413.

8. Sciacco M, Prelle A, Comi GP, et al. Retrospective study of a large population of patients affected with mitochondrial disorders: clinical, morphological and molecular genetic evaluation. J Neurol. 2001;248(9):778-88

9. Yamashita S, Nishino I, Nonaka I, Goto Y. Genotype and phenotype analyses in 136 patients with single large-scale mitochondrial DNA deletions. J Hum Genet. 2008;53(7):598-606.

10. Richardson C, Smith T, Schaefer A, Turnbull D, Griffiths P. Ocular motility findings in chronic progressive external ophthalmoplegia. Eye. 2005;19(3):258-63.

11. Walker M, Taylor RW, Turnbull DM. Mitochondrial diabetes. Diabet Med. 2005;22 Suppl 4:18-20.

12. Kornblum C, Broicher R, Walther E, et al. Cricopharyngeal achalasia is a common cause of dysphagia in patients with mtDNA deletions. Neurology. 2001;56(10):1409-12.
13. Danta G, Hilton RC, Lynch PG. Chronic progressive external ophthalmoplegia. Brain. 1975;98(3):473-92.

14. Caballero PE, Candela MS, Alvarez CI, Tejerina AA. Chronic progressive external ophthalmoplegia: a report of 6 cases and a review of the literature. Neurologist. 2007;13(1):33-6.

15. Razmara A, Duckles SP, Krause DN, Procaccio V. Estrogen suppresses brain mitochondrial oxidative stress in female and male rats. Brain Res. 2007;1176:71-81.

16. Brierley EJ, Johnson MA, Lightowlers RN, James OF, Turnbull DM. Role of mitochondrial DNA mutations in human aging: implications for the central nervous system and muscle. Ann Neurol. 1998;43(2):217-23.

17. Schaefer AM, Blakely EL, Griffiths PG, Turnbull DM, Taylor RW. Ophthalmoplegia due to mitochondrial DNA disease: the need for genetic diagnosis. Muscle Nerve. 2005;32(1):104-7.

18. Carlow TJ, Depper MH, Orrison WW Jr. MR of extraocular muscles in chronic progressive external ophthalmoplegia. AJNR Am J Neuroradiol. 1998;19(1):95-9.

19. Ozkan SB, Soylev MF, Karaman ZC, Kir E, Durak I. The evaluation of chronic progressive external ophthalmoplegia with computerized tomography. Int Ophthalmol. 2001;24(1):37-9.

20. Dinges WL, Witherspoon SR, Itani KM, Garg A, Peterson DM. Blepharoptosis and external ophthalmoplegia associated with long-term antiretroviral therapy. Clin Infect Dis. 2008;47(6): 845-52.

21. Fraunfelder FW, Richards AB. Diplopia, blepharoptosis, and ophthalmoplegia and 3-hydroxy-3-methyl-glutaryl-CoA reductase inhibitor use. Ophthalmology. 2008;115(12):2282-5.

22. Kirkman MA, Yu-Wai-Man P, Korsten A, et al. Gene-environment interactions in leber hereditary optic neuropathy. Brain. 2009 Sep;132(Pt 9):2317-26.

23. Masayesva BG, Mambo E, Taylor RJ, et al. Mitochondrial DNA content increase in response to cigarette smoking. Cancer Epidemiol Biomarkers Prev. 2006;15(1):19-24.

24. Korenaga M, Okuda M, Otani K, Wang T, Li Y, Weinman SA. Mitochondrial dysfunction in hepatitis C. J Clin Gastroenterol. 2005;39(4 Suppl 2):S162-6.

25. Smoking prevalence in British Columbia [homepage on the internet]. Ipsos-Reid. 2003 Sept 2 [cited 2010 Jun 17]. Available from: http://www.health.gov.bc.ca/tobacco/pdf/ipsos.pdf.

26. Wong T, Lee SS. Hepatitis C: a review for primary care physicians. CMAJ. 2006; 174(5):649-59. 